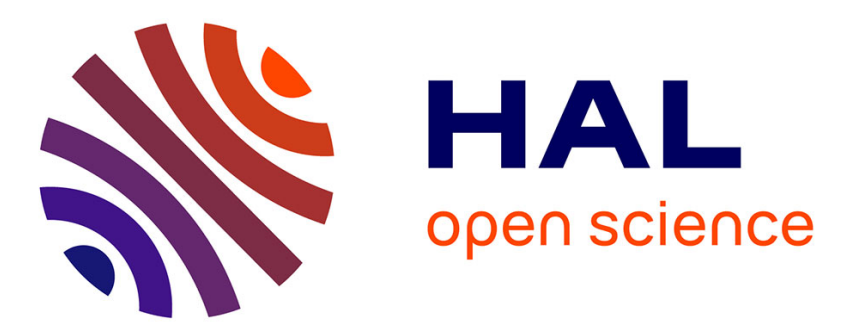

\title{
First study of the interference between initial and final state radiation at the $\mathrm{Z}$ resonance
}

\author{
P. Abreu, W. Adam, T. Adye, E. Agasi, I. Ajinenko, R. Aleksan, G D.
} Alekseev, R. Alemany, P P. Allport, S. Almehed, et al.

\section{- To cite this version:}

P. Abreu, W. Adam, T. Adye, E. Agasi, I. Ajinenko, et al.. First study of the interference between initial and final state radiation at the $\mathrm{Z}$ resonance. Zeitschrift für Physik $\mathrm{C}$ Particles and Fields, 1996, 72, pp.31-38. 10.1007/s002880050220 . in2p3-00002445

\section{HAL Id: in2p3-00002445 \\ https://hal.in2p3.fr/in2p3-00002445}

Submitted on 10 Feb 1999

HAL is a multi-disciplinary open access archive for the deposit and dissemination of scientific research documents, whether they are published or not. The documents may come from teaching and research institutions in France or abroad, or from public or private research centers.
L'archive ouverte pluridisciplinaire HAL, est destinée au dépôt et à la diffusion de documents scientifiques de niveau recherche, publiés ou non, émanant des établissements d'enseignement et de recherche français ou étrangers, des laboratoires publics ou privés. 


\title{
First Study of the Interference between Initial and Final State Radiation at the $\mathrm{Z}$ Resonance
}

\author{
DELPHI Collaboration
}

\begin{abstract}
The interference between initial and final state radiation in the process $e^{+} e^{-} \rightarrow \mu^{+} \mu^{-}$at $\sqrt{s} \approx \mathrm{M}_{\mathrm{Z}}$ has been studied by measuring the forward-backward asymmetry as a function of the acoplanarity angle between the final state muons. The interference is expected to be sensitive to the space-time separation of the initial and final state radiation. The measured asymmetry distribution has been compared to theoretical predictions using the KORALZ generator, with and without $\mathcal{O}(\alpha)$ interference. The magnitude of the interference between initial and final state radiation was found to be of the order predicted and to follow the expected distribution. Using the theoretical predictions, a value of

$$
\Gamma_{\mathrm{Z}}=2.50 \pm 0.21 \text { (stat.) } \pm 0.06 \text { (syst.) } \mathrm{GeV} \text {. }
$$

has been extracted. The interpretation of this result is discussed. There is an additional uncertainty in the estimate of $\Gamma_{Z}$ from as yet uncalculated higher order interference terms. By assuming a value of $\Gamma_{Z}$ consistent with the world average, the data were used to estimate the size of these uncalculated corrections.
\end{abstract}


P.Abreu ${ }^{21}$, W.Adam ${ }^{50}$, T.Adye ${ }^{37}$, E.Agasi $^{31}$, I.Ajinenko ${ }^{42}$, R.Aleksan ${ }^{39}$, G.D.Alekseev ${ }^{16}$, R.Alemany ${ }^{49}$, P.P.Allport ${ }^{22}$, S.Almehed ${ }^{24}$, U.Amaldi ${ }^{9}$, S.Amato ${ }^{47}$, A.Andreazza ${ }^{28}$, M.L.Andrieux ${ }^{14}$, P.Antilogus ${ }^{9}$, W-D.Apel ${ }^{17}$, Y.Arnoud ${ }^{39}$, B.Asman ${ }^{44}$, J-E.Augustin ${ }^{25}$, A.Augustinus ${ }^{9}$, P.Baillon ${ }^{9}$, P.Bambade ${ }^{19}$, R.Barate $^{14}$,

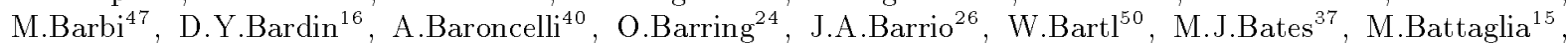
M.Baubillier $^{23}$, J.Baudot ${ }^{39}$, K-H.Becks ${ }^{52}$, M.Begalli ${ }^{6}$, P.Beilliere ${ }^{8}$, Yu.Belokopytov ${ }^{9,53}$, K.Belous ${ }^{42}$, A.C.Benvenuti $^{5}$, M.Berggren ${ }^{47}$, D.Bertrand ${ }^{2}$, F.Bianchi ${ }^{45}$, M.Bigi ${ }^{45}$, M.S.Bilenky ${ }^{16}$, P.Billoir ${ }^{23}$, D.Bloch ${ }^{10}$, M.Blume $^{52}$, S.Blyth ${ }^{35}$, T.Bolognese ${ }^{39}$, M.Bonesini ${ }^{28}$, W.Bonivento ${ }^{28}$, P.S.L.Booth ${ }^{22}$, G.Borisov ${ }^{42}$, C.Bosio $^{40}$, S.Bosworth $^{35}$, O.Botner ${ }^{48}$, E.Boudinov ${ }^{31}$, B.Bouquet ${ }^{19}$, C.Bourdarios ${ }^{9}, \quad$ T.J.V.Bowcock ${ }^{22}$, M.Bozzo ${ }^{13}$, P.Branchini $^{40}$, K.D.Brand ${ }^{36}$, T.Brenke ${ }^{52}$, R.A.Brenner ${ }^{15}$, C.Bricman ${ }^{2}$, L.Brillault ${ }^{23}$, R.C.A.Brown ${ }^{9}$, P.Bruckman $^{18}$, J-M.Brunet ${ }^{8}$, L.Bugge ${ }^{33}$, T.Buran ${ }^{33}$, T.Burgsmueller ${ }^{52}$, P.Buschmann ${ }^{52}$, A.Buys ${ }^{9}$, S.Cabrera ${ }^{49}$,

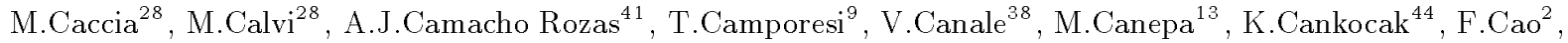
F.Carena $^{9}$, L.Carroll ${ }^{22}$, C.Caso ${ }^{13}$, M.V.Castillo Gimenez ${ }^{49}$, A.Cattai ${ }^{9}$, F.R.Cavallo ${ }^{5}$, L.Cerrito $^{38}$, V.Chabaud $^{9}$, Ph.Charpentier $^{9}$, L.Chaussard ${ }^{25}$, J.Chauveau ${ }^{23}$, P.Checchia ${ }^{36}$, G.A.Chelkov ${ }^{16}$, M.Chen ${ }^{2}$, R.Chierici ${ }^{45}$, P.Chliapnikov $^{42}$, P.Chochula ${ }^{7}$, V.Chorowicz ${ }^{9}$, J.Chudoba ${ }^{30}$, V.Cindro ${ }^{43}$, P.Collins ${ }^{9}$, J.L.Contreras ${ }^{19}$, R.Contri ${ }^{13}$, E.Cortina ${ }^{49}$, G.Cosme ${ }^{19}$, F.Cossutti ${ }^{46}$, H.B.Crawley ${ }^{1}$, D.Crennell ${ }^{37}$, G.Crosetti ${ }^{13}$, J.Cuevas Maestro $^{34}$, S.Czellar ${ }^{15}$, E.Dahl-Jensen ${ }^{29}$, J.Dahm ${ }^{52}$, B.Dalmagne ${ }^{19}$, M.Dam ${ }^{29}$, G.Damgaard ${ }^{29}$, P.D.Dauncey ${ }^{37}$, M.Davenport ${ }^{9}$, W.Da Silva ${ }^{23}$, C.Defoix ${ }^{8}$, A.Deghorain ${ }^{2}$, G.Della Ricca ${ }^{46}$, P.Delpierre ${ }^{27}$, N.Demaria $^{35}$, A.De Angelis ${ }^{9}$, W.De Boer ${ }^{17}$, S.De Brabandere ${ }^{2}$, C.De Clercq ${ }^{2}$ C.De La Vaissiere ${ }^{23}$, B.De Lotto ${ }^{46}$, A.De Min ${ }^{36}$, L.De Paula ${ }^{47}$, C.De Saint-Jean ${ }^{39}$, H.Dijkstra ${ }^{9}$, L.Di Ciaccio ${ }^{38}$, F.Djama $^{10}$, J.Dolbeau $^{8}$, M.Donszelmann ${ }^{9}$, K.Doroba ${ }^{51}$, M.Dracos ${ }^{10}$, J.Drees ${ }^{52}$, K.-A.Drees ${ }^{52}$, M.Dris ${ }^{32}$, Y.Dufour ${ }^{9}$, D.Edsall $^{1}$, R.Ehret ${ }^{17}$, G.Eigen ${ }^{4}$, T.Ekelof ${ }^{48}$, G.Ekspong ${ }^{44}$, M.Elsing ${ }^{52}$, J-P.Engel ${ }^{10}$, N.Ershaidat ${ }^{23}$, B.Erzen $^{43}$, M.Espirito Santo ${ }^{21}$, E.Falk ${ }^{24}$, D.Fassouliotis ${ }^{32}$, M.Feindt ${ }^{9}$, A.Ferrer ${ }^{49}$, T.A.Filippas ${ }^{32}$, A.Firestone ${ }^{1}$, P.-A.Fischer ${ }^{10}$, H.Foeth ${ }^{9}$, E.Fokitis ${ }^{32}$, F.Fontanelli ${ }^{13}$, F.Formenti ${ }^{9}$, B.Franek ${ }^{37}$, P.Frenkiel ${ }^{8}$, D.C.Fries ${ }^{17}$, A.G.Frodesen ${ }^{4}$, F.Fulda-Quenzer ${ }^{19}$, J.Fuster ${ }^{49}$, A.Galloni ${ }^{22}$, D.Gamba ${ }^{45}$, M.Gandelman ${ }^{6}$, C.Garcia $^{49}$, J.Garcia $^{41}$, C.Gaspar ${ }^{9}$, U.Gasparini ${ }^{36}$, Ph.Gavillet ${ }^{9}$, E.N.Gazis ${ }^{32}$, D.Gele ${ }^{10}$, J-P.Gerber ${ }^{10}$, L.Gerdyukov $^{42}$, M.Gibbs ${ }^{22}$, R.Gokieli ${ }^{51}$, B.Golob ${ }^{43}$, G.Gopal $^{37}$, L.Gorn ${ }^{1}$, M.Gorski $^{51}$, Yu.Gouz ${ }^{45,53}$, V.Gracco ${ }^{13}$, E.Graziani ${ }^{40}$, G.Grosdidier $^{19}$, K.Grzelak ${ }^{51}$, S.Gumenyuk ${ }^{28,53}$, P.Gunnarsson ${ }^{44}$, M.Gunther ${ }^{48}$, J.Guy ${ }^{37}$, F.Hahn ${ }^{9}$, S.Hahn ${ }^{52}$, A.Hallgren $^{48}$, K.Hamacher ${ }^{52}$, W.Hao ${ }^{31}$, F.J.Harris ${ }^{35}$, V.Hedberg ${ }^{24}$, R.Henriques ${ }^{21}$, J.J.Hernandez ${ }^{49}$, P.Herquet $^{2}$, H.Herr ${ }^{9}$, T.L.Hessing ${ }^{35}$, E.Higon ${ }^{49}$, H.J.Hilke ${ }^{9}$, T.S.Hill ${ }^{1}$, S-O.Holmgren ${ }^{44}$, P.J.Holt ${ }^{35}$, D.Holthuizen ${ }^{31}$, S.Hoorelbeke ${ }^{2}$, M.Houlden ${ }^{22}$, J.Hrubec ${ }^{50}$, K.Huet ${ }^{2}$, K.Hultqvist ${ }^{44}$, J.N.Jackson $^{22}$, R.Jacobsson ${ }^{44}$, P.Jalocha ${ }^{18}$, R.Janik ${ }^{7}$, Ch.Jarlskog ${ }^{24}$, G.Jarlskog ${ }^{24}$, P.Jarry ${ }^{39}$, B.Jean-Marie ${ }^{19}$, E.K.Johansson ${ }^{44}$, L.Jonsson ${ }^{24}$, P.Jonsson ${ }^{24}$, C.Joram ${ }^{9}$, P.Juillot ${ }^{10}$, M.Kaiser ${ }^{17}$, F.Kapusta ${ }^{23}$, K.Karafasoulis ${ }^{11}$, M.Karlsson $^{44}$, E.Karvelas ${ }^{11}$, S.Katsanevas ${ }^{3}$, E.C.Katsoufis ${ }^{32}$, R.Keranen ${ }^{4}$, Yu.Khokhlov ${ }^{42}$, B.A.Khomenko ${ }^{16}{ }^{\text {, }}$ N.N.Khovanski ${ }^{16}$, B.King ${ }^{22}$, N.J.Kjaer ${ }^{29}$, H.Klein ${ }^{9}$, A.Klovning ${ }^{4}$, P.Kluit ${ }^{31}$, B.Koene ${ }^{31}$, P.Kokkinias ${ }^{11}$, M.Koratzinos $^{9}$, K.Korcyl ${ }^{18}$, C.Kourkoumelis ${ }^{3}$, O.Kouznetsov ${ }^{13,16}$, P.-H.Kramer ${ }^{52}$, M.Krammer ${ }^{50}$, C.Kreuter $^{17}$, I.Kronkvist $^{24}$, Z.Krumstein ${ }^{16}$, W.Krupinski ${ }^{18}$, P.Kubinec ${ }^{7}$, W.Kucewicz ${ }^{18}$, K.Kurvinen ${ }^{15}$, C.Lacasta $^{49}$, I.Laktineh $^{25}$, S.Lamblot ${ }^{23}$, J.W.Lamsa ${ }^{1}$, L.Lanceri ${ }^{46}$, D.W.Lane ${ }^{1}$, P.Langefeld ${ }^{52}$, I.Last ${ }^{22}$, J-P.Laugier $^{39}$, R.Lauhakangas $^{15}$, G.Leder ${ }^{50}$, F.Ledroit ${ }^{14}$, V.Lefebure ${ }^{2}$, C.K.Legan ${ }^{1}$, R.Leitner ${ }^{30}$, Y.Lemoigne ${ }^{39}$, J.Lemonne ${ }^{2}$, G.Lenzen $^{52}$, V.Lepeltier ${ }^{19}$, T.Lesiak ${ }^{36}$, D.Liko ${ }^{50}$, R.Lindner ${ }^{52}$, A.Lipniacka ${ }^{36}$, I.Lippi ${ }^{36}$, B.Loerstad $^{24}$, J.G.Loken ${ }^{35}$, J.M.Lopez ${ }^{41}$, D.Loukas ${ }^{11}$, P.Lutz ${ }^{39}$, L.Lyons ${ }^{35}$, J.MacNaughton ${ }^{50}$, G.Maehlum ${ }^{17}$, A.Maio ${ }^{21}$, V.Malychev $^{16}$, F.Mandl ${ }^{50}$, J.Marco ${ }^{41}$, R.Marco ${ }^{41}$, B.Marechal ${ }^{47}$, M.Margoni ${ }^{36}$, J-C.Marin ${ }^{9}$, C.Mariotti ${ }^{40}$, A.Markou $^{11}$, T.Maron ${ }^{52}$, C.Martinez-Rivero ${ }^{41}$, F.Martinez-Vidal ${ }^{49}$, S.Marti i Garcia ${ }^{49}$, J.Masik ${ }^{30}$, F.Matorras $^{41}$, C.Matteuzzi ${ }^{9}$, G.Matthiae ${ }^{38}$, M.Mazzucato ${ }^{36}$, M.Mc Cubbin ${ }^{9}$, R.Mc Kay ${ }^{1}$, R.Mc Nulty ${ }^{22}$, J.Medbo $^{48}$, M.Merk ${ }^{31}$, C.Meroni ${ }^{28}$, S.Meyer ${ }^{17}$, W.T.Meyer ${ }^{1}$, M.Michelotto ${ }^{36}$, E.Migliore ${ }^{45}$, L.Mirabito ${ }^{25}$, W.A.Mitaroff ${ }^{50}$, U.Mjoernmark ${ }^{24}$, T.Moa ${ }^{44}$, R.Moeller ${ }^{29}$, K.Moenig ${ }^{9}$, M.R.Monge ${ }^{13}$, P.Morettini ${ }^{13}$, H.Mueller ${ }^{17}$, L.M.Mundim ${ }^{6}$, W.J.Murray ${ }^{37}$, B.Muryn ${ }^{18}$, G.Myatt ${ }^{35}$, F.Naraghi ${ }^{14}$, F.L.Navarria ${ }^{5}$, S.Navas ${ }^{49}$, K.Nawrocki ${ }^{51}$, P.Negri ${ }^{28}$, W.Neumann ${ }^{52}$, N.Neumeister ${ }^{50}$, R.Nicolaidou ${ }^{3}$, B.S.Nielsen ${ }^{29}$, M.Nieuwenhuizen ${ }^{31}$, V.Nikolaenko ${ }^{10}$, P.Niss ${ }^{44}$, A.Nomerotski ${ }^{36}$, A.Normand ${ }^{35}$, M.Novak ${ }^{12}$, W.Oberschulte-Beckmann ${ }^{17}$, V.Obraztsov ${ }^{42}$, A.G.Olshevski ${ }^{16}$, A.Onofre ${ }^{21}$, R.Orava ${ }^{15}$, K.Osterberg ${ }^{15}$, A.Ouraou ${ }^{39}$, P.Paganini ${ }^{19}$, M.Paganoni $^{9}$, P.Pages $^{10}$, H.Palka ${ }^{18}$, Th.D.Papadopoulou ${ }^{32}$, K.Papageorgiou ${ }^{11}$, L.Pape ${ }^{9}$, C.Parkes ${ }^{35}$,

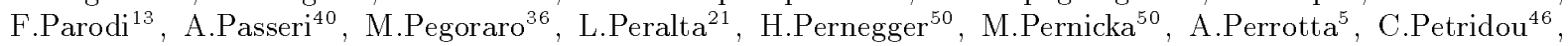
A.Petrolini ${ }^{13}$, M.Petrovyck ${ }^{28,53}$, H.T.Phillips ${ }^{37}$, G.Piana ${ }^{13}$, F.Pierre ${ }^{39}$, M.Pimenta ${ }^{21}, \quad$ M.Pindo $^{28}$, S.Plaszczynski $^{19}$, O.Podobrin ${ }^{17}$, M.E.Pol ${ }^{6}$, G.Polok ${ }^{18}$, P.Poropat ${ }^{46}$, V.Pozdniakov ${ }^{16}$, M.Prest ${ }^{46}$, P.Privitera ${ }^{38}$, N.Pukhaeva ${ }^{16}$, A.Pullia ${ }^{28}$, D.Radojicic ${ }^{35}$, S.Ragazzi ${ }^{28}$, H.Rahmani ${ }^{32}$, P.N.Ratoff ${ }^{20}$, A.L.Read ${ }^{33}$, M.Reale $^{52}$, P.Rebecchi $^{19}$, N.G.Redaelli ${ }^{28}$, M.Regler ${ }^{50}$, D.Reid ${ }^{9}$, P.B.Renton ${ }^{35}$, L.K.Resvanis ${ }^{3}$, F.Richard ${ }^{19}$, J.Richardson ${ }^{22}$, J.Ridky $^{12}$, G.Rinaudo ${ }^{45}$, I.Ripp ${ }^{39}$, A.Romero ${ }^{45}$, I.Roncagliolo ${ }^{13}$, P.Ronchese ${ }^{36}$, L.Roos ${ }^{14}$, E.I.Rosenberg ${ }^{1}$, E.Rosso $^{9}$, P.Roudeau ${ }^{19}$, T.Rovelli ${ }^{5}$, W.Ruckstuhl ${ }^{31}$, V.Ruhlmann-Kleider ${ }^{39}$, A.Ruiz ${ }^{41}$, K.Rybicki ${ }^{18}$, A.Rybin $^{42}$, H.Saarikko ${ }^{15}$, Y.Sacquin ${ }^{39}$, A.Sadovsky ${ }^{16}$, G.Sajot $^{14}$, J.Salt ${ }^{49}$, J.Sanchez ${ }^{26}$, M.Sannino ${ }^{13}$, M.Schimmelpfennig ${ }^{17}$, H.Schneider ${ }^{17}$, U.Schwickerath ${ }^{17}$, M.A.E.Schyns ${ }^{52}$, G.Sciolla ${ }^{45}$, F.Scuri ${ }^{46}$, P.Seager ${ }^{20}$, Y.Sedykh ${ }^{16}$, A.M.Segar ${ }^{35}$,

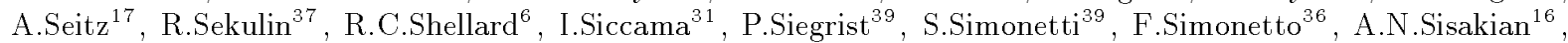
B.Sitar ${ }^{7}$, T.B.Skaali ${ }^{33}$, G.Smadja ${ }^{25}$, N.Smirnov ${ }^{42}$, O.Smirnova ${ }^{24}$, G.R.Smith ${ }^{37}$, O.Solovianov ${ }^{42}$, R.Sosnowski ${ }^{51}$, D.Souza-Santos ${ }^{6}$, T.Spassov ${ }^{21}$, E.Spiriti ${ }^{40}$, P.Sponholz ${ }^{52}$, S.Squarcia ${ }^{13}$, C.Stanescu $^{40}$, S.Stapnes ${ }^{33}$, I.Stavitski ${ }^{36}$, F.Stichelbaut $^{9}$, A.Stocchi ${ }^{19}$, J.Strauss ${ }^{50}$, R.Strub ${ }^{10}$, B.Stugu ${ }^{4}$, M.Szczekowski ${ }^{51}$, M.Szeptycka ${ }^{51}$, T.Tabarelli ${ }^{28}$, 
J.P.Tavernet ${ }^{23}$, O.Tchikilev ${ }^{42}$, A.Tilquin ${ }^{27}$, J.Timmermans ${ }^{31}$, L.G.Tkatchev ${ }^{16}$, T.Todorov ${ }^{10}$, D.Z.Toet $^{31}$, A.Tomaradze $^{2}$, B.Tome ${ }^{21}$, A.Tonazzo ${ }^{28}$, L.Tortora ${ }^{40}$, G.Transtromer ${ }^{24}$, D.Treille ${ }^{9}$, W.Trischuk ${ }^{9}$, G.Tristram ${ }^{8}$, A.Trombini ${ }^{19}$, C.Troncon ${ }^{28}$, A.Tsirou ${ }^{9}$, M-L.Turluer ${ }^{39}$, I.A.Tyapkin ${ }^{16}$, M.Tyndel ${ }^{37}, \quad$ S.Tzamarias ${ }^{22}$, B.Ueberschaer $^{52}$, O.Ullaland ${ }^{9}$, V.Uvarov ${ }^{42}$, G.Valenti ${ }^{5}$, E.Vallazza ${ }^{9}$, G.W.Van Apeldoorn ${ }^{31}$, P.Van Dam ${ }^{31}$, W.K.Van Doninck ${ }^{2}$, J.Van Eldik ${ }^{31}$, N.Vassilopoulos ${ }^{35}$, G.Vegni ${ }^{28}$, L.Ventura ${ }^{36}$, W.Venus ${ }^{37}$, F.Verbeure ${ }^{2}$, M.Verlato $^{36}$, L.S.Vertogradov ${ }^{16}$, D.Vilanova ${ }^{39}$, P.Vincent ${ }^{25}$, L.Vitale ${ }^{46}$, E.Vlasov ${ }^{42}$, A.S.Vodopyanov $^{16}$, V.Vrba $^{12}$, H.Wahlen ${ }^{52}$, C.Walck ${ }^{44}$, F.Waldner ${ }^{46}$, M.Weierstall ${ }^{52}$, P.Weilhammer ${ }^{9}$, C.Weiser ${ }^{17}$, A.M.Wetherell ${ }^{9}$, D.Wicke $^{52}$, J.H.Wickens ${ }^{2}$, M.Wielers ${ }^{17}$, G.R.Wilkinson ${ }^{35}$, W.S.C.Williams ${ }^{35}$, M.Winter ${ }^{10}$, M.Witek $^{18}$, K.Woschnagg $^{48}$, K.Yip ${ }^{35}$, O.Yushchenko ${ }^{42}$, F.Zach ${ }^{25}$, A.Zaitsev ${ }^{42}$, A.Zalewska ${ }^{18}$, P.Zalewski ${ }^{51}$, D.Zavrtanik ${ }^{43}$, E.Zevgolatakos ${ }^{11}$, N.I.Zimin ${ }^{16}$, M.Zito ${ }^{39}$, D.Zontar ${ }^{43}$, R.Zuberi ${ }^{35}$, G.C.Zucchelli ${ }^{44}$, G.Zumerle ${ }^{36}$

\footnotetext{
${ }^{1}$ Ames Laboratory and Department of Physics, Iowa State University, Ames IA 50011, USA

${ }^{2}$ Physics Department, Univ. Instelling Antwerpen, Universiteitsplein 1, B-2610 Wilrijk, Belgium and IIHE, ULB-VUB, Pleinlaan 2, B-1050 Brussels, Belgium

and Faculté des Sciences, Univ. de l'Etat Mons, Av. Maistriau 19, B-7000 Mons, Belgium

${ }^{3}$ Physics Laboratory, University of Athens, Solonos Str. 104, GR-10680 Athens, Greece

${ }^{4}$ Department of Physics, University of Bergen, Allégaten 55, N-5007 Bergen, Norway

${ }^{5}$ Dipartimento di Fisica, Università di Bologna and INFN, Via Irnerio 46, I-40126 Bologna, Italy

${ }^{6}$ Centro Brasileiro de Pesquisas Físicas, rua Xavier Sigaud 150, RJ-22290 Rio de Janeiro, Brazil and Depto. de Física, Pont. Univ. Católica, C.P. 38071 RJ-22453 Rio de Janeiro, Brazil and Inst. de Física, Univ. Estadual do Rio de Janeiro, rua São Francisco Xavier 524, Rio de Janeiro, Brazil ${ }^{7}$ Comenius University, Faculty of Mathematics and Physics, Mlynska Dolina, SK-84215 Bratislava, Slovakia

${ }^{8}$ Collège de France, Lab. de Physique Corpusculaire, IN2P3-CNRS, F-75231 Paris Cedex 05, France

${ }^{9} \mathrm{CERN}, \mathrm{CH}-1211$ Geneva 23, Switzerland

${ }^{10}$ Centre de Recherche Nucléaire, IN2P3 - CNRS/ULP - BP20, F-67037 Strasbourg Cedex, France

${ }^{11}$ Institute of Nuclear Physics, N.C.S.R. Demokritos, P.O. Box 60228, GR-15310 Athens, Greece

${ }^{12}$ FZU, Inst. of Physics of the C.A.S. High Energy Physics Division, Na Slovance 2, 180 40, Praha 8, Czech Republic

${ }^{13}$ Dipartimento di Fisica, Università di Genova and INFN, Via Dodecaneso 33, I-16146 Genova, Italy

${ }^{14}$ Institut des Sciences Nucléaires, IN2P3-CNRS, Université de Grenoble 1, F-38026 Grenoble Cedex, France

${ }^{15}$ Research Institute for High Energy Physics, SEFT, P.O. Box 9, FIN-00014 Helsinki, Finland

${ }^{16}$ Joint Institute for Nuclear Research, Dubna, Head Post Office, P.O. Box 79, 101000 Moscow, Russian Federation

${ }^{17}$ Institut für Experimentelle Kernphysik, Universität Karlsruhe, Postfach 6980, D-76128 Karlsruhe, Germany

${ }^{18}$ Institute of Nuclear Physics and University of Mining and Metalurgy, Ul. Kawiory 26a, PL-30055 Krakow, Poland

${ }^{19}$ Université de Paris-Sud, Lab. de l'Accélérateur Linéaire, IN2P3-CNRS, Bât. 200, F-91405 Orsay Cedex, France

${ }^{20}$ School of Physics and Materials, University of Lancaster, Lancaster LA1 4YB, UK

${ }^{21}$ LIP, IST, FCUL - Av. Elias Garcia, 14-1 ${ }^{\circ}$, P-1000 Lisboa Codex, Portugal

${ }^{22}$ Department of Physics, University of Liverpool, P.O. Box 147, Liverpool L69 3BX, UK

${ }^{23}$ LPNHE, IN2P3-CNRS, Universités Paris VI et VII, Tour 33 (RdC), 4 place Jussieu, F-75252 Paris Cedex 05, France

${ }^{24}$ Department of Physics, University of Lund, Sölvegatan 14, S-22363 Lund, Sweden

${ }^{25}$ Université Claude Bernard de Lyon, IPNL, IN2P3-CNRS, F-69622 Villeurbanne Cedex, France

${ }^{26}$ Universidad Complutense, Avda. Complutense s/n, E-28040 Madrid, Spain

${ }^{27}$ Univ. d'Aix - Marseille II - CPP, IN2P3-CNRS, F-13288 Marseille Cedex 09, France

${ }^{28}$ Dipartimento di Fisica, Università di Milano and INFN, Via Celoria 16, I-20133 Milan, Italy

${ }^{29}$ Niels Bohr Institute, Blegdamsvej 17, DK-2100 Copenhagen 0, Denmark

${ }^{30}$ NC, Nuclear Centre of MFF, Charles University, Areal MFF, V Holesovickach 2, 180 00, Praha 8, Czech Republic

${ }^{31}$ NIKHEF-H, Postbus 41882, NL-1009 DB Amsterdam, The Netherlands

${ }^{32}$ National Technical University, Physics Department, Zografou Campus, GR-15773 Athens, Greece

${ }^{33}$ Physics Department, University of Oslo, Blindern, N-1000 Oslo 3, Norway

${ }^{34}$ Dpto. Fisica, Univ. Oviedo, C/P. Pérez Casas, S/N-33006 Oviedo, Spain

${ }^{35}$ Department of Physics, University of Oxford, Keble Road, Oxford OX1 3RH, UK

${ }^{36}$ Dipartimento di Fisica, Università di Padova and INFN, Via Marzolo 8, I-35131 Padua, Italy

${ }^{37}$ Rutherford Appleton Laboratory, Chilton, Didcot OX11 OQX, UK

${ }^{38}$ Dipartimento di Fisica, Università di Roma II and INFN, Tor Vergata, I-00173 Rome, Italy

${ }^{39}$ Centre d'Etudes de Saclay, DSM/DAPNIA, F-91191 Gif-sur-Yvette Cedex, France

${ }^{40}$ Istituto Superiore di Sanità, Ist. Naz. di Fisica Nucl. (INFN), Viale Regina Elena 299, I-00161 Rome, Italy

${ }^{41}$ Instituto de Fisica de Cantabria (CSIC-UC), Avda. los Castros, S/N-39006 Santander, Spain, (CICYT-AEN93-0832)

${ }^{42}$ Inst. for High Energy Physics, Serpukov P.O. Box 35, Protvino, (Moscow Region), Russian Federation

${ }^{43} \mathrm{~J}$. Stefan Institute and Department of Physics, University of Ljubljana, Jamova 39, SI-61000 Ljubljana, Slovenia

${ }^{44}$ Fysikum, Stockholm University, Box 6730, S-113 85 Stockholm, Sweden

${ }^{45}$ Dipartimento di Fisica Sperimentale, Università di Torino and INFN, Via P. Giuria 1, I-10125 Turin, Italy

${ }^{46}$ Dipartimento di Fisica, Università di Trieste and INFN, Via A. Valerio 2, I-34127 Trieste, Italy and Istituto di Fisica, Università di Udine, I-33100 Udine, Italy

${ }^{47}$ Univ. Federal do Rio de Janeiro, C.P. 68528 Cidade Univ., Ilha do Fundão BR-21945-970 Rio de Janeiro, Brazil

${ }^{48}$ Department of Radiation Sciences, University of Uppsala, P.O. Box 535, S-751 21 Uppsala, Sweden

${ }^{49}$ IFIC, Valencia-CSIC, and D.F.A.M.N., U. de Valencia, Avda. Dr. Moliner 50, E-46100 Burjassot (Valencia), Spain

${ }^{50}$ Institut für Hochenergiephysik, Österr. Akad. d. Wissensch., Nikolsdorfergasse 18, A-1050 Vienna, Austria

${ }^{51}$ Inst. Nuclear Studies and University of Warsaw, Ul. Hoza 69, PL-00681 Warsaw, Poland

${ }^{52}$ Fachbereich Physik, University of Wuppertal, Postfach 100 127, D-42097 Wuppertal 1, Germany

${ }^{53}$ On leave of absence from IHEP Serpukhov
} 


\section{Introduction}

This paper presents the first study of the interference between initial state radiation (ISR) and final state radiation (FSR) in the process $e^{+} e^{-} \rightarrow \mu^{+} \mu^{-}$at the peak energy of the $Z$ resonance. This interference is a probe of the space-time structure of the process and is sensitive to the $\mathrm{Z}$ lifetime, $\tau_{\mathrm{Z}}$, and thus to the $\mathrm{Z}$ width, $\Gamma_{\mathrm{Z}}$. The data used were taken by the DELPHI Collaboration at LEP between 1992 and 1994.

In the process $e^{+} e^{-} \rightarrow \mu^{+} \mu^{-}$at centre of mass energies close to the $\mathrm{Z}$ mass, $\sqrt{s} \approx \mathrm{M}_{\mathrm{Z}}$, the relative importance of the interference between initial and final state radiation depends on the restrictions placed on the phase space available to the emitted photons $[1,2]$. For loose experimental cuts, the effect of radiative interference is predicted to be very small. The $\mathcal{O}(\alpha)$ difference between the cross sections calculated with and without interference is proportional to $(\alpha / \pi)\left(\Gamma_{\mathrm{Z}} / \mathrm{M}_{\mathrm{Z}}\right)^{2}$. For the forward-backward asymmetry [1], $A_{\mathrm{FB}}$, the difference $\delta A_{\mathrm{FB}}^{\mathrm{int}}$ is defined by:

$$
\delta A_{\mathrm{FB}}^{\mathrm{int}}=\frac{\left(\sigma_{f}+\delta_{f}\right)-\left(\sigma_{b}+\delta_{b}\right)}{\left(\sigma_{f}+\delta_{f}\right)+\left(\sigma_{b}+\delta_{b}\right)}-\frac{\sigma_{f}-\sigma_{b}}{\sigma_{f}+\sigma_{b}} \approx \frac{\delta_{f}-\delta_{b}}{\sigma_{f}+\sigma_{b}}
$$

where $\sigma_{f(b)}$ are the forward (backward) cross sections calculated without interference to all known orders, and $\delta_{f(b)}$ are the changes to the forward (backward) cross sections due to $\mathcal{O}(\alpha)$ interference. The magnitude of $\delta A_{\mathrm{FB}}^{\mathrm{int}}$ is proportional to $(\alpha / \pi)\left(\Gamma_{\mathrm{Z}} / \mathrm{M}_{\mathrm{Z}}\right)$. This is much smaller than the correction to the Born level $A_{\mathrm{FB}}$ introduced by $\mathcal{O}(\alpha)$ noninterference QED terms which, on the peak of the $\mathrm{Z}$ resonance, are comparable in size to the Born asymmetry. However, if appropriate tight cuts are placed on the photon phase space then the effects of radiative interference can become large enough to be observed experimentally [3]. For example, the size of $\delta A_{\mathrm{FB}}^{\mathrm{int}}$ depends strongly on the cut on the maximum energy of photons; the lower the maximum energy accepted, the greater the effect of the interference.

A physical explanation as to why interference should be more important for low photon energy is as follows [2,3]. Initial state radiation is associated with the annihilation of the incoming $e^{+} e^{-}$pair, final state radiation with the creation of the outgoing $\mu^{+} \mu^{-}$pair. The time separation between the initial and final state radiation is determined by the lifetime of the $\mathrm{Z}$, which is related to the width of the $\mathrm{Z}$ by the uncertainty principle, $\tau_{\mathrm{Z}}=\hbar / \Gamma_{\mathrm{Z}}$. The uncertainty in the time at which a photon of energy $E_{\gamma}$ is created is likewise given by $\hbar / E_{\gamma}$. So for photons with energies less than approximately $\Gamma_{\mathrm{Z}}$, the initial and final state radiation become indistinguishable and can interfere strongly. This argument suggests that examining $A_{\mathrm{FB}}$ as a function of cuts on the photon energy should provide a different method of measuring $\Gamma_{\mathrm{Z}}$, which is traditionally obtained from lineshape measurements [4]. This space-time picture is complementary to a momentumspace analysis of the interaction, assuming that the process can be treated within the framework of standard Quantum Field Theory. The space-time picture gives a qualitative, intuitive, description of the process. In practice, however, the quantitative variation of $\delta A_{\mathrm{FB}}^{\mathrm{int}}$ with $\Gamma_{\mathrm{Z}}$ has been calculated using momentum-space techniques.

The most direct experimental approach would be to search for photons in the reaction $e^{+} e^{-} \rightarrow \mu^{+} \mu^{-}$which have energies less than about $2.5 \mathrm{GeV}$, and to measure $A_{\mathrm{FB}}$ for events where such photons are seen. Both initial and final state radiation can give rise to muons which are not back-to-back. An alternative approach is to therefore replace the measurement of the photon energy with that of the acoplanarity or acollinearity ${ }^{\dagger}$ of the

\footnotetext{
${ }^{\dagger}$ The acoplanarity angle is a measure of the acollinearity between the two particles, $i$ and $j$, in the plane perpendicular to the two incident beams, the $r-\phi$ plane, and is defined as: $\phi_{a c o p}=|180-| \phi_{i}-\phi_{j}||$. The acollinearity angle for two
} 
muons in the final state. Using the acoplanarity was found to give a higher statistical precision than using either the acollinearity or the photon energy.

\section{Theoretical Predictions}

The generator KORALZ [5] was used to predict the forward-backward asymmetry of muon pairs as a function of acoplanarity for a range of values of $\Gamma_{\mathrm{Z}}$. These predictions were then compared with the data. Figure 1 shows the predicted acoplanarity as a function of the photon energy. It shows that the probability of having a large acoplanarity between the muons in an event increases with the energy of the emitted photon. Therefore, restricting the acoplanarity indirectly restricts the photon energy, and should make manifest the interference of initial and final state radiation.

The treatment of the interference between ISR and FSR in KORALZ is based on calculations performed in momentum-space which include only one radiated photon. There have been no theoretical calculations of higher order interference terms as yet. The version of KORALZ used for this study provides two alternative sets of calculations. In the first, only $\mathcal{O}(\alpha)$ interference and non-interference QED corrections are computed. In the second, higher order non-interference corrections including exponentiation are calculated, but the radiative interference terms are omitted. These higher order calculations can be combined with the $\mathcal{O}(\alpha)$ interference corrections but, without the corresponding interference terms, their inclusion does not necessarily constitute a genuine improvement to the theoretical model. Therefore, the data were first compared to the prediction of KORALZ with strictly $\mathcal{O}(\alpha)$ QED corrections, in the part of phase space where radiative interference corrections are dominant. The higher order non-interference terms were then used to obtain an estimate of the importance of the unknown interference terms. Figure 2(a) shows the predictions of the generator including and excluding radiative interference in the simulation.

In the branch of KORALZ which includes only $\mathcal{O}(\alpha)$ QED corrections to Born level terms, a soft photon cut-off is implemented during event generation. Photons with energy less than this cut-off do not have full kinematic information generated, and do not give rise to non-zero acoplanarities between final state muons. This unphysical cut-off cannot be made arbitrarily small and was a potential source of bias in the data analysis. To control this bias, predictions were made for two values of the soft photon cut-off. As can be seen in figure $2(\mathrm{a})$, the predictions in the region of acoplanarity below $0.63^{\circ}$ depended on the cut-off. Therefore, data were not compared to theoretical predictions for acoplanarities below $0.63^{\circ}$. Also, no comparison was made above $10^{\circ}$, due to the low number of events

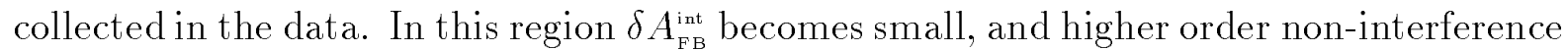
corrections need to be included to obtain reasonable agreement between the theoretical predictions and the data. A systematic uncertainty (see Table 1, below) due to the soft photon cut-off was estimated for the comparisons in the range of acoplanarity from $0.63^{\circ}$ to $10^{\circ}$. The standard value for the cut-off was taken to be $0.0025 \sqrt{s} / 2$, the lowest value acceptable in the generator. About $50 \%$ of the generated events were classified as soft-photon events with the standard cut-off parameter. The computation of higher order radiative interference terms would be a significant improvement to the theoretical predictions.

particles with momenta $\mathbf{p}_{m}$ and $\mathbf{p}_{n}$ is defined by: $\cos \theta_{a c o l}=-\mathbf{p}_{m} \cdot \mathbf{p}_{n} /\left|\mathbf{p}_{m}\right|\left|\mathbf{p}_{n}\right|$, and is a measure of the acollinearity in 3 dimensions. Throughout this paper angles are measured in degrees using the DELPHI coordinate system in which the $z$-axis points along the direction of the electron beam and the $x$-axis points towards the centre of the LEP storage ring. 


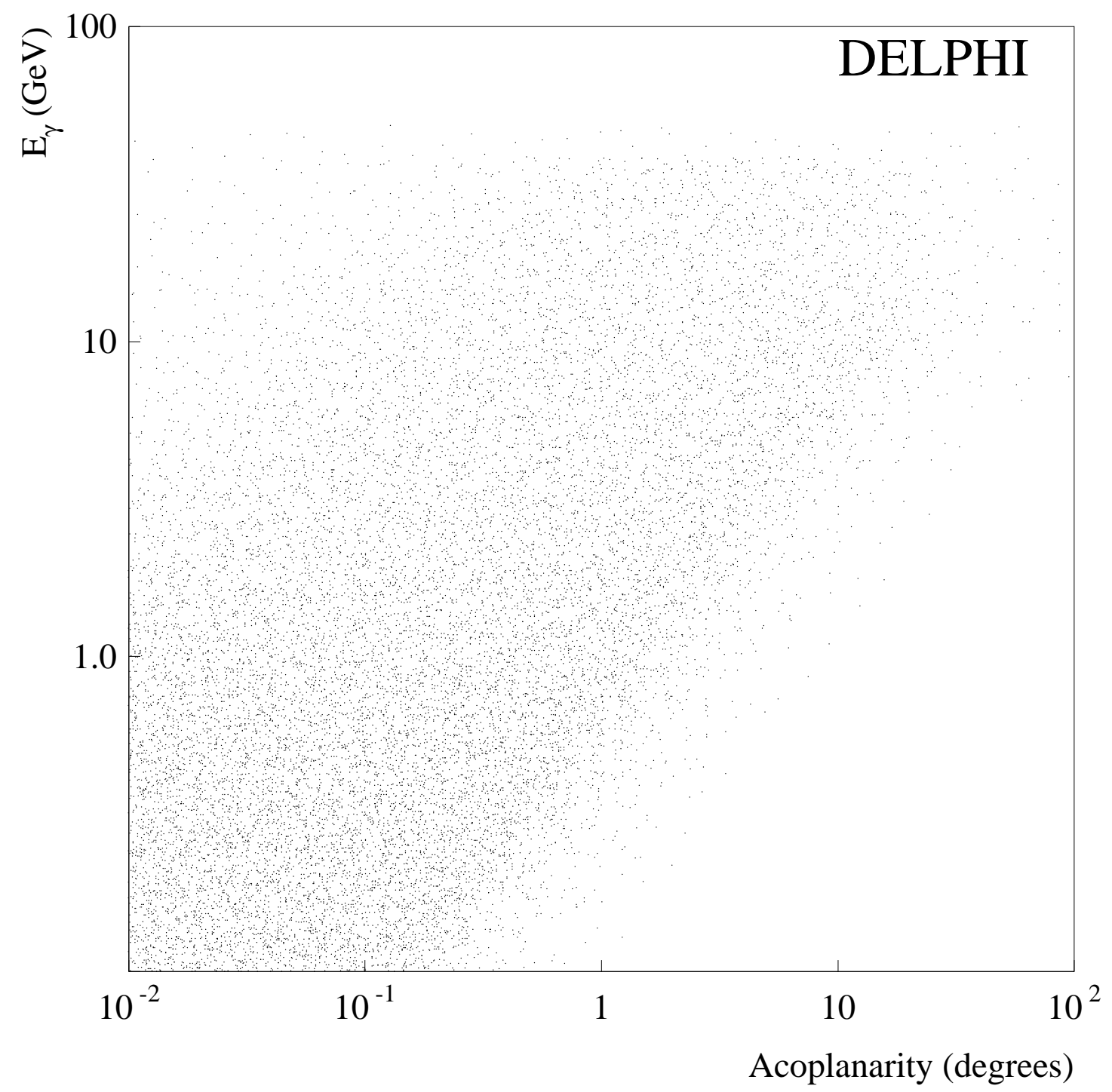

Figure 1: Acoplanarity vs the photon energy in events generated using KORALZ with $\mathcal{O}(\alpha)$ QED radiation. 


\section{DELPHI}
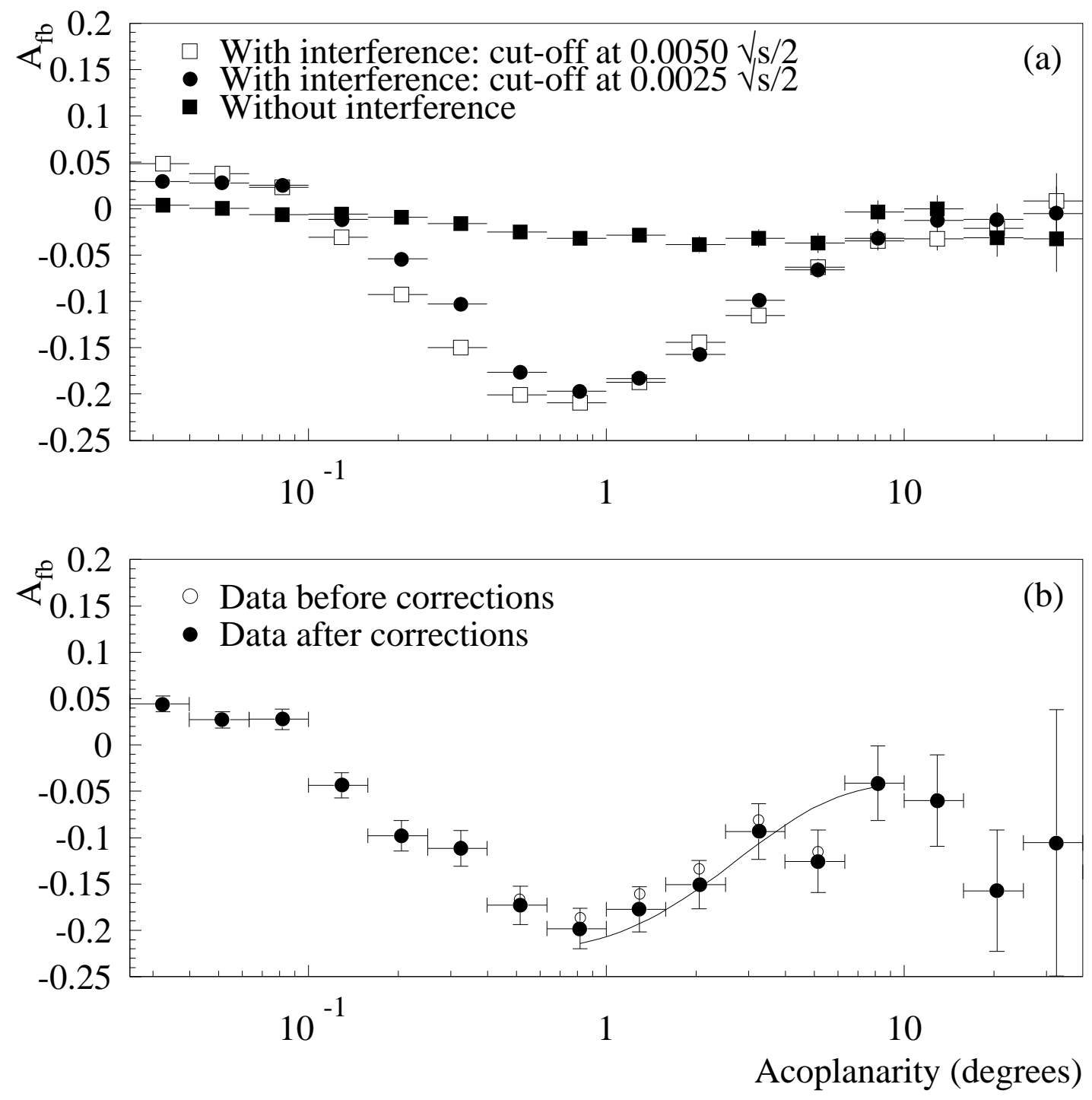

Figure 2: The distribution of $A_{F B}$, as a function of acoplanarity plotted on a logarithmic scale, for: (a) theoretical predictions from KORALZ assuming $\Gamma_{\mathrm{Z}}=2.497 \mathrm{GeV}$, with and without the effects of the ISR-FSR interference and also showing the sensitivity to the soft photon cut-off; (b) all selected $\mu^{+} \mu^{-}$events, before and after corrections for backgrounds as discussed in the text. The solid line shows the theoretical predictions for the fitted value of $\Gamma_{\mathrm{Z}}$ after smearing the $\phi$ values of the generated muons as described in the text. 
In the absence of radiative interference, the forward-backward asymmetry as a function of acoplanarity depends on the variation of $A_{\mathrm{FB}}$ with the centre of mass energy $\sqrt{s^{\prime}}$ of the hard scattering process. This asymmetry is dominated by the interference between the exchange of a $Z$ or a photon between the initial and final states. The value of $\sqrt{s^{\prime}}$ is determined by the energy of photons radiated from the incoming particles. For acoplanarities in the range $0.63^{\circ}$ to $10.0^{\circ}$, the photons are more likely to be from FSR than from ISR. The probability of producing an ISR photon with a high component of momentum in the $r$ - $\phi$ plane is suppressed, compared to FSR, by the energy dependence of the Z exchange. However, FSR does not lower the centre of mass energy of the hard scattering. Hence, without radiative interference, $A_{\mathrm{FB}}$ is expected, and is predicted by detailed calculations, to be approximately independent of acoplanarity.

The shape of the predicted asymmetry distribution after including radiative interference can be understood as follows. For low acoplanarities, the predictions of the event generator are unreliable due to the soft photon cut-off: many of the events in this region have photons with energies below this cut-off and hence do not have proper kinematic information. For intermediate acoplanarities, the events predominantly contain photons of energies comparable to the width of the $Z$, and are therefore expected to be sensitive to radiative interference. Large acoplanarities correspond to high energy photons which produce little interference effect.

To examine the accuracy of KORALZ, the predictions were compared with the semianalytical calculations of ZFITTER [6], which calculates the cross-sections in the forward and backward hemispheres as a function of cut-offs on the invariant mass or acollinearity of the $\mu^{+} \mu^{-}$pair. The calculations as a function of acollinearity contain approximations making them unsuitable for comparison with KORALZ. As a function of the invariant mass, the predicted shift in the asymmetry introduced by radiative interference agreed with the value predicted by KORALZ within $0.25 \%$ on average. This was taken to be the precision of KORALZ as a function of acoplanarity. It was concluded that the predictions of KORALZ were satisfactory for the purposes of this study. This small uncertainty on $\delta A_{\mathrm{FB}}^{\text {int }}$ was considered to be a second source of systematic error on the parameters extracted below.

\section{The Data}

The data used for this analysis were collected by the DELPHI detector [7] in 1992, 1993 and 1994. The 1993 data used were from the peak point of the LEP energy scan only, the 1994 data were those collected up to the shutdown in October. To select $\mu^{+} \mu^{-}$events, criteria were applied which are similar to those described in more detail in [8]. For the analysis described here, the following cuts were used. There had to be at least 2 charged particles found in each event, the momentum of each of the two fastest charged particles had to be larger than $5 \mathrm{GeV}$, and the radial momentum ${ }^{\ddagger}$ had to be larger than $28 \mathrm{GeV}$. The polar angle of the fastest negative particle with respect to the incoming electron had to be between $11^{\circ}$ and $169^{\circ}$ and the acollinearity of the two fastest particles had to be be smaller than $20^{\circ}$. Both of the two fastest particles in each event had to be identified as muons, based on the association of hits in muon chambers or on the observation of energy deposits in the electromagnetic and hadronic calorimeters consistent with a minimum ionising particle. The background from cosmic ray muons

\footnotetext{
${ }^{\ddagger}$ The radial momentum is defined as $p_{\text {rad }}=\sqrt{\left(p_{1}^{2}+p_{2}^{2}\right) / 2}$ where $p_{1,2}$ are the momenta of the first and second fastest charged particles in each event.
} 
was suppressed by cuts on the distance of closest approach of the muons to the point at which the incoming $e^{+}$and $e^{-}$beams collided. A further cut was applied to remove muons that passed into regions of the detector where there was evidence for a bias in the forward-backward asymmetry. Events were rejected if the azimuthal angle, $\phi$, of either muon, modulo $60^{\circ}$, was between $28^{\circ}$ and $32^{\circ}$. This corresponds to the boundaries between the sectors of DELPHI's principal tracking chamber, the Time Projection Chamber. The asymmetry of events in these regions was not statistically compatible with the rest of the data. A sample of about $97,000 \mu^{+} \mu^{-}$events was selected with these criteria.

The forward-backward asymmetry in each acoplanarity bin was calculated by counting the number of events with negatively charged muons in the forward and backward hemispheres with respect to the incident electron direction. For small intervals of acoplanarity, the angular distribution is not well represented by a simple $1+\cos ^{2} \theta+R \cos \theta$ shape, although this form is accurate to a very good approximation for the angular distribution integrated over all acoplanarities. Detector inefficiencies were taken into account by weighting each event by the inverse of the detection efficiency. This was found to vary as a function the polar angle $\theta$ and was determined by comparing the data with the theoretically predicted $\cos \theta$ distribution integrated over all acoplanarities and folded about $\theta=90^{\circ}$, which has a simple $1+\cos ^{2} \theta$ shape.

Other possible detector biases and backgrounds in the selected sample were studied as follows.

The response of the DELPHI detector could be different for positive and negative muons. The asymmetry of the data was measured using both the distribution of negatively charged muons and that of positively charged muons in the forward and backward hemispheres. The two separate distributions were consistent with one another. To compare with theoretical predictions, an average was taken of these two distributions. To evaluate a systematic error, fits were also made using the negatively and positively charged distributions separately. The difference between the results of these fits was negligible.

Figure 2(b) shows the charge-averaged forward-backward asymmetry as a function of acoplanarity for all selected $\mu^{+} \mu^{-}$candidates, before and after corrections for misidentified $\tau^{+} \tau^{-}$events and cosmic rays were applied. These corrections are discussed below.

Mismeasurement of the azimuthal angle, $\phi$, of muons could move events from their true acoplanarity bin to another, thereby mixing events of different intrinsic asymmetries and changing the measured value of $A_{\mathrm{FB}}$ from the underlying physical value. The absence of any significant net offset in acoplanarity in the events selected from the data was verified by checking that the mean value of $\left|\phi_{1}-\phi_{2}\right|$ was statistically consistent with $180^{\circ}$. Here $\phi_{1}$ and $\phi_{2}$ are the azimuthal angles of the two muons, with $\phi_{1}$ that of the fastest muon. To account for the measurement errors in the data, an algorithm was used to smear the $\phi$ angles of the muons generated by KORALZ. The algorithm applied a Gaussian smearing, the width of which depended on the polar angle of the muons. The widths of the Gaussians were tuned to bring the acoplanarity distributions of the generated $\mu^{+} \mu^{-}$events into reasonable agreement with the data. These integrated distributions are predicted to be insensitive to the details of radiative interference. To investigate the sensitivity of the simulation to the choice of the smearing parameters, an alternative method was used in which the azimuthal angles of all muons, irrespective of polar angle, were smeared by a single Gaussian of width $0.022^{\circ}$ which corresponded to the mean measurement precision. This led to a systematic shift in the value of $\Gamma_{\mathrm{Z}}$ extracted in this study of $0.03 \mathrm{GeV}$, see Table 1 below. The asymmetry distribution was most sensitive to the smearing at acoplanarities below $0.63^{\circ}$. As already mentioned, this region was 
anyway not used in the fits below because the $\mathcal{O}(\alpha)$ predictions were unreliable there due to the soft photon cut-off.

In approximately $0.5 \%$ of all events, the two fastest particles had the same apparent charge due to mismeasurement, Nearly $50 \%$ of these like-sign muons had $|\cos \theta|$ larger than 0.8. Often the momentum of one of the muons was measured to be unphysically high. Excluding or including the like-sign events produced a small but significant change in the measured asymmetry distribution as a function of acoplanarity. The asymmetry of like-sign events was determined by attempting to resolve which muon had been badly reconstructed and forcing the sign of its charge to be opposite to that assigned. It was assumed that the observed acoplanarity distribution for these events, which was peaked around $1^{\circ}$, was the result of the poor measurement of $\phi$ of one or both of the identified muons, and that the underlying acoplanarity distribution of like-sign events was similar to that of well measured muons. It was found that the asymmetry of the like-sign events was consistent with being constant as a function of acoplanarity, and the average value of $A_{\mathrm{FB}}$ obtained was $0.028 \pm 0.056$. For the fits of the theory to the data described below, the like-sign events were removed from the data. Using generated events it was estimated that rejecting a random sample of events with a $\cos \theta$ distribution similar to the like-sign events in the data leads to a systematic shift in the value of $\Gamma_{Z}$ extracted in this study of $0.01 \mathrm{GeV}$. This was taken to be the size of the systematic error arising from the treatment of the like-sign events.

Events where one or more of the muons had an uncertainty on the $\phi$ measurement larger than $5^{\circ}$ accounted for $0.04 \%$ of the data sample. Like the like-sign events, these events were not well modelled by the smearing algorithm described above. Therefore they were excluded when comparing the theoretical predictions to the data. Including them shifted the extracted value of $\Gamma_{\mathrm{Z}}$ by $0.01 \mathrm{GeV}$, more than expected either from purely statistical fluctuations or from the increase in efficiency resulting from their inclusion.

Other possible detector biases, investigated using $\mu^{+} \mu^{-}$events generated by DYMU3 [9] and passed through the DELPHI detector simulation package DELSIM [7], were found to be negligible. The DYMU3 generator does not include radiative interference, therefore the $A_{\mathrm{FB}}$ distribution was approximately constant as a function of acoplanarity, which made it insensitive to the $\phi$ resolution. After applying the same selection criteria to both the generated and reconstructed events, no significant differences were found in the $A_{\mathrm{FB}}$ distributions.

The largest background in the $\mu^{+} \mu^{-}$event sample came from misidentified $\tau^{+} \tau^{-}$events. From Monte Carlo simulation, this background was found to be $(0.83 \pm 0.20) \%$ of all events. A study using $\tau^{+} \tau^{-}$events generated by KORALZ indicated that interference effects were not significant in the asymmetry distributions as a function of acoplanarity for those $\tau^{+} \tau^{-}$decays which pass the $\mu^{+} \mu^{-}$selection criteria. Therefore the $\tau^{+} \tau^{-}$background was investigated using a full simulation of the detector's response to $\tau^{+} \tau^{-}$events, in which radiative interference was not included. The acoplanarity distribution is shown in figure 3 for all simulated $e^{+} e^{-} \rightarrow \tau^{+} \tau^{-}$events selected as $\mu^{+} \mu^{-}$events, normalized to all selected $\mu^{+} \mu^{-}$events. The full simulation predictions for the asymmetry as a function of acoplanarity were fitted to a constant value of $A_{\mathrm{FB}}$. The value of the $\tau^{+} \tau^{-}$background asymmetry extracted was $0.010 \pm 0.032$. The data were corrected for the $\tau^{+} \tau^{-}$background, using this central value and the acoplanarity distribution of misidentified simulated $\tau^{+} \tau^{-}$events. The uncertainty on the shift in $A_{\mathrm{FB}}$ in each acoplanarity bin of the data was $\pm 30 \%$ of the mean shift on average. This uncertainty was taken as a systematic error in fits of the theoretical predictions of the generator to the data and gave the largest shift in the value of $\Gamma_{\mathrm{Z}}, 0.05 \mathrm{GeV}$. 
The background from cosmic ray muons in the selected sample was estimated, using a sample of cosmic ray events, to be $(0.11 \pm 0.01) \%$, but gave negligible systematic error on the parameters extracted in this analysis. Further backgrounds from two photon events and misidentified Bhabha events have been shown to be negligible.

\section{Comparison of Theoretical Predictions to the Data}

To compare theoretical predictions to data, $\mu^{+} \mu^{-}$events were generated using KORALZ, with and without interference between initial and final state radiation. An ansatz largely independent of the Standard Model was used, in which $\Gamma_{\mathrm{Z}}$ and $\sin ^{2} \theta_{\mathrm{W}}^{\mathrm{eff}}$ were both taken as independent parameters. Throughout, $\sin ^{2} \theta_{\mathrm{W}}^{\mathrm{eff}}$ was taken to be 0.2318 , consistent with the value reported in [4]. This parameter sets the effective coupling constants of the $\mathrm{Z}$ to fermions. The kinematical cuts that were used for data were applied to the generated events. As mentioned above, the only detector effect that was found to be important to include in the simulation was the smearing of the $\phi$ of each muon to reproduce the measurement precision in DELPHI.

To show that the effect of interference between initial state and final state radiation was present in the data, $\chi^{2}$ fits were made between the theoretical predictions of the generator, with and without radiative interference, and the data, assuming a total $\mathrm{Z}$ width of $2.497 \mathrm{GeV}$ [4]. The known sources of systematic uncertainty were taken into account by repeating the fits for different levels of background, different choices of smearing parameterisation and like-sign contributions, etc. To test the sensitivity of the fits to various assumptions excluding radiative interference from KORALZ, fits were made to purely $\mathcal{O}(\alpha)$ predictions and to predictions including higher order corrections. In all cases the fits were performed for 6 acoplanarity bins between $0.63^{\circ}$ and $10^{\circ}$.

Assuming no interference between initial and final state radiation, the data and the theoretical predictions were incompatible, see figure 2. The $\chi^{2}$ for the fit between predictions and data was never less than 108 for 6 degrees of freedom. If the interference term was included, the data were in agreement with the theoretical expectations. For the variations in assumptions mentioned above, the probability that the data were compatible with random fluctuations from the predicted distribution was never found to be smaller than $61 \%$. The assumptions that the observed asymmetry distribution could be explained either by purely initial state radiation or by purely final state radiation were incompatible with the data. Fits resulted in $\chi^{2}$ values of 186 and 388, respectively, for the two hypotheses.

The data, therefore, show a strong indication of interference between initial and final state radiation, and of the magnitude predicted by the theoretical model. 


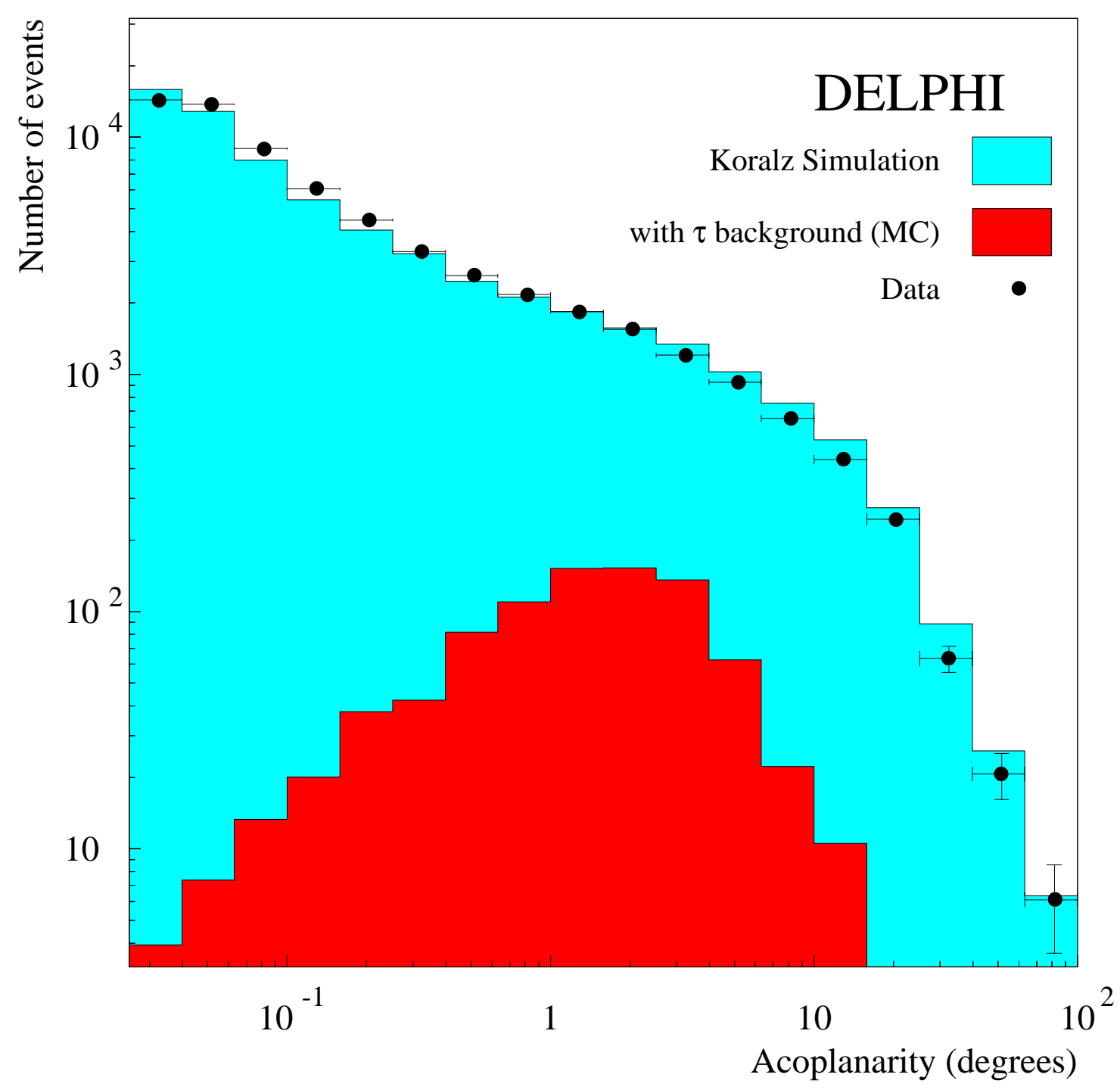

Figure 3: The acoplanarity distribution for selected simulated $e^{+} e^{-} \rightarrow \tau^{+} \tau^{-}$events, compared to $e^{+} e^{-} \rightarrow \mu^{+} \mu^{-}$events simulated by KORALZ, and $e^{+} e^{-} \rightarrow \mu^{+} \mu^{-}$events identified in the data. The number of $e^{+} e^{-} \rightarrow \tau^{+} \tau^{-}$events has been normalised to the total number of events in the data. 


\begin{tabular}{|l|l|l|l|}
\hline Source & Assumption & $\Gamma_{\mathrm{Z}}(\mathrm{GeV})$ & Systematic error \\
\hline \hline Soft photon cut-off & $0.0050 \sqrt{s} / 2$ & 2.51 & $\pm 0.01 \mathrm{GeV}$ \\
\hline$\delta A_{\mathrm{FB}}^{\text {int }}($ ZFITTER/KORALZ) & \pm 0.0005 & 2.51 & $\pm 0.01 \mathrm{GeV}$ \\
\hline \hline$\phi$ smearing & $\begin{array}{l}\text { Single } \\
\text { Gaussian }\end{array}$ & 2.47 & $\pm 0.03 \mathrm{GeV}$ \\
\hline Like-signs & included & 2.49 & $\pm 0.01 \mathrm{GeV}$ \\
\hline $\begin{array}{l}\text { Events with large } \\
\text { errors on } \phi\end{array}$ & included & 2.49 & $\pm 0.01 \mathrm{GeV}$ \\
\hline$\tau$ background & +0.042 & 2.55 & \\
asymmetry & -0.021 & 2.45 & $\pm 0.05 \mathrm{GeV}$ \\
\hline \hline Total & & & $\pm 0.06 \mathrm{GeV}$ \\
\hline
\end{tabular}

Table 1: Sources of systematic error.

\section{$5 \quad$ Fit to $\Gamma_{\mathrm{Z}}$}

The amplitude of the change in $A_{\mathrm{FB}}$ as a function of acoplanarity resulting from interference between ISR and FSR is expected to depend on the width of the Z. Figure 4 shows the expected variation in $\delta A_{\mathrm{FB}}^{\mathrm{int}}$ as a function of acoplanarity, for five different values of $\Gamma_{Z}$.

Fits were made between the predictions and the data, for acoplanarities from $0.63^{\circ}$ to $10^{\circ}$ and for 9 values of $\Gamma_{\mathrm{Z}}$ between $0.5 \mathrm{GeV}$ and $4.5 \mathrm{GeV}$. The resulting $\chi^{2}$ distribution was parameterised by a polynomial in $\Gamma_{Z}$, and the value of the total $\mathrm{Z}$ width obtained was

$$
\Gamma_{\mathrm{Z}}=2.50 \pm 0.21 \text { (stat.) } \mathrm{GeV}
$$

with a $\chi^{2}$ of 2.7 for 5 degrees of freedom. The component of the statistical error from the simulation statistics was $0.05 \mathrm{GeV}$. A systematic error on $\Gamma_{\mathrm{Z}}$ was calculated from the variations in the central value of $\Gamma_{Z}$ from the different sources of bias, both in the theoretical predictions and in the data, as discussed in sections 2 and 3 respectively. Table 1 shows the individual contributions. Including the systematic error,

$$
\Gamma_{\mathrm{Z}}=2.50 \pm 0.21 \text { (stat.) } \pm 0.06 \text { (syst.) } \mathrm{GeV} \text {. }
$$

As mentioned above, interference terms have not been calculated at orders higher than $\mathcal{O}(\alpha)$. However, using the event generator KORALZ it is possible to include the higher order non-interference terms. Without the corresponding interference corrections this does not necessarily constitute a genuine improvement to the theory, but can be used to obtain an estimate of the importance of the higher order interference corrections.

The $\chi^{2}$ for a comparison of the theoretical predictions, including known higher order non-interference corrections, with the data, over the same range of acoplanarities as above, was 64.7 for 6 degrees of freedom. This was calculated assuming the $\mathrm{Z}$ width to be $2.497 \mathrm{GeV}$. Taking $\Gamma_{\mathrm{Z}}$ to be a free parameter, the best fit was $-1.1 \mathrm{GeV}$ below that obtained with purely $\mathcal{O}(\alpha)$ radiative corrections. This shift can be understood as follows. For acoplanarities in the range of interest, the higher order non-interference corrections reduce the predicted cross section as a function of acoplanarity. This reflects the fact that configurations with several low energy photons are preferred to those with 


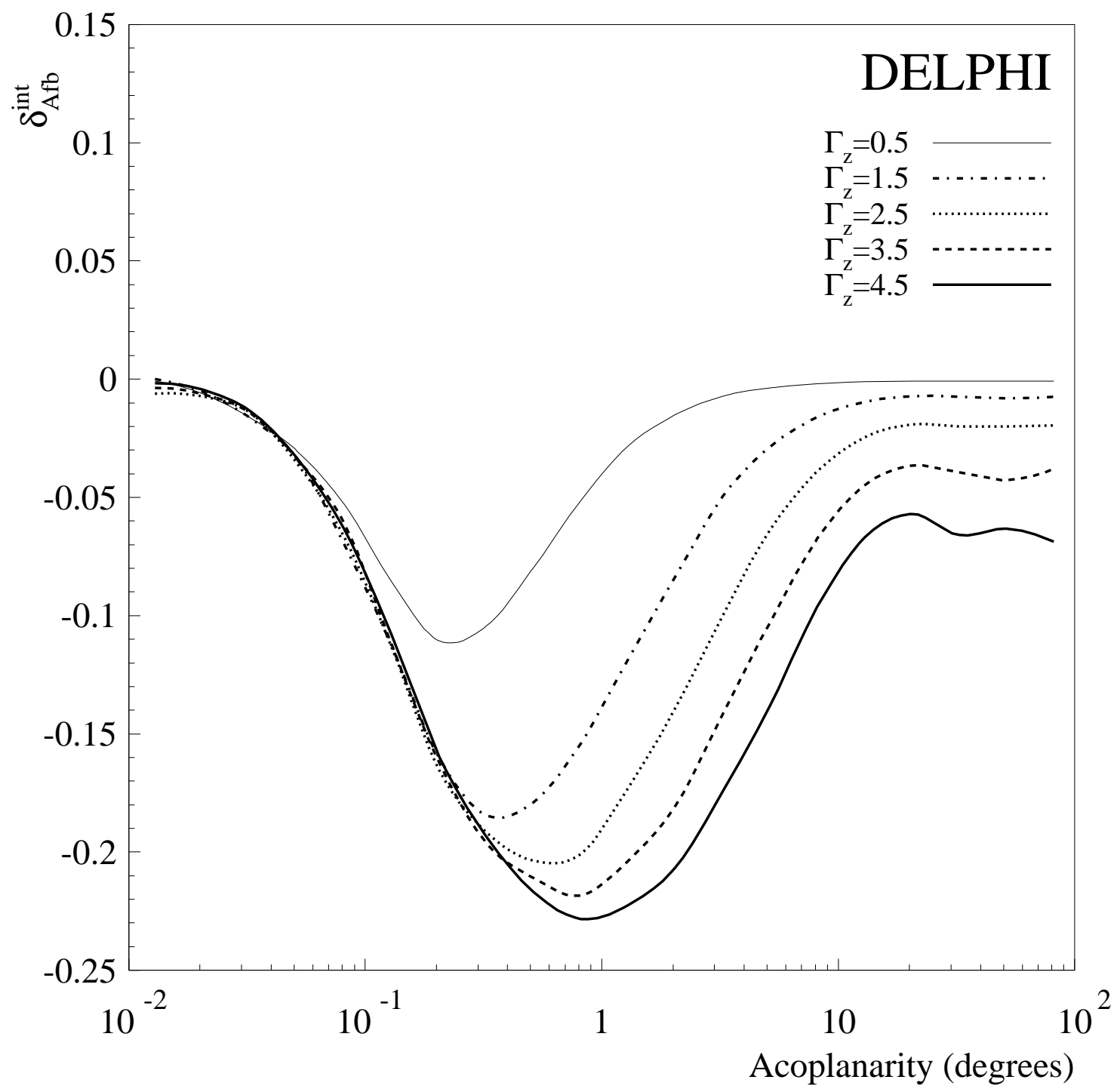

Figure 4: The KORALZ prediction for the change in $A_{F B}$ due to radiative interference, as a function of acoplanarity, for five different values of $\Gamma_{\mathrm{Z}}$ between 0.5 and $4.5 \mathrm{GeV}$. Small statistical fluctuations resulting from the number of events generated have not been smoothed out. 
single high energy photons. Thus the denominator in the expression for $A_{\mathrm{FB}}$ is reduced, but the numerator, which is dominated by the $\mathcal{O}(\alpha)$ interference terms, is essentially unchanged. Therefore, for fixed $\Gamma_{\mathrm{Z}}$, the predictions for $A_{\mathrm{FB}}$ are larger than at $\mathcal{O}(\alpha)$. The discrepancy between the data and the predictions was used to gauge the size of the higher order interference corrections by assuming that $\Gamma_{\mathrm{Z}}$ was equal to the value measured in lineshape studies, and that the whole discrepancy was due to the missing interference corrections. The change to $A_{\mathrm{FB}}$ due to higher order radiative interference was estimated by subtracting the predicted forward-backward asymmetry distribution including higher order non-interference corrections from the data distribution, which was assumed to contain all higher order corrections. This correction was found to be approximately $60 \%$ of the $\mathcal{O}(\alpha)$ correction, and positive, over the range of acoplanarities used in this analysis. This result can be checked by explicit theoretical calculations.

\section{Conclusions and Discussion}

To investigate the interference between initial and final state radiation, the forwardbackward asymmetry of $\mu^{+} \mu^{-}$events has been studied as a function of the acoplanarity of the muons. It has been shown that this effect is expected to become significant only for tight cuts on the photon phase space in $e^{+} e^{-} \rightarrow \mu^{+} \mu^{-}$events at $\sqrt{s} \approx \mathrm{M}_{\mathrm{Z}}$. The size of these interference effects agrees with the predictions of the theoretical model implemented in KORALZ, which includes radiative interference at $\mathcal{O}(\alpha)$. The possibility that there is no interference between initial and final state radiation is excluded by the data, to a high degree of confidence.

The size of the radiative interference is expected to depend on the lifetime of the $\mathrm{Z}$ or equivalently, according to quantum mechanics, on $\Gamma_{\mathrm{Z}}$. A fit to the width of the $Z$, using that part of the data for which the $\mathcal{O}(\alpha)$ QED corrections are not highly sensitive to the soft photon cut-off in KORALZ, gave a value of

$$
\Gamma_{\mathrm{Z}}=2.50 \pm 0.21 \text { (stat.) } \pm 0.06 \text { (syst.) GeV } .
$$

In the generator KORALZ, the effects of radiative interference included are based on momentum-space calculations and are parameterised in terms of $\Gamma_{Z}$. The effect of radiative interference is described naturally in the space-time picture discussed above. This picture suggests that hypothetical violations of the quantum mechanical relationship $\Gamma_{Z}=\hbar / \tau_{Z}$ could make the value of $\Gamma_{Z}$ determined in this analysis inconsistent with the value determined from lineshape studies, $2.497 \mathrm{GeV}$ [4]. Experimentally this ratio was found to be

$$
\frac{\Gamma_{Z}^{\text {lineshape }}}{\Gamma_{Z}^{\text {interference }}}=1.00 \pm 0.09
$$

which is compatible with the expected ratio of unity. Therefore, using the time-energy uncertainty relationship, the lifetime of the $\mathrm{Z}$ determined by this analysis is

$$
\tau_{\mathrm{Z}}=(2.63 \pm 0.24) \times 10^{-25} \mathrm{~s} .
$$

For further discussion on the possible interpretations of these results, see [3]. There is an additional uncertainty in the above results arising from as yet uncalculated higher order interference terms. The most significant improvement to this analysis would be the inclusion of higher order interference terms into the theoretical predictions. These corrections were estimated to be approximately $60 \%$ of the $\mathcal{O}(\alpha)$ corrections, in the range of acoplanarities studied in this analysis, and positive. 


\section{Acknowledgements}

We would like to thank Z. Was for many helpful discussions in the interpretation of the data using KORALZ. We are greatly indebted to our technical collaborators and to the funding agencies for their support in building and operating the DELPHI detector, and to the members of the CERN-SL Division for the excellent performance of the LEP collider.

\section{References}

[1] M. Böhm \& W. Hollik, "Forward-backward Asymmetries", in "Z Physics at LEP 1", (ed: G. Altarelli, R. Kleiss, C. Verzegnassi), CERN 89-08 (1989) 203;

Z. Was \& S. Jadach, Phys. Rev. D41 (1990) 1425;

S. Jadach, J.H. Kuhn \& Z. Was, Z. Phys. C38 (1988) 609.

[2] S. Jadach \& Z. Was, Phys. Lett. B219 (1989) 103.

[3] S. Jadach \& Z. Was, CERN-TH 7232/94 (1994).

[4] D. Schaile, Proceedings of the XXVII International Conference on High Energy Physics, Glasgow, Scotland, UK, 20-27 July 1994, Editors P.J.. Bussey and I.G. Knowles, IOP publishing (1995) 27.

[5] S. Jadach, B.F.L. Ward \& Z. Was, Comp. Phys. Comm. 79 (1994) 503.

[6] D. Bardin et al , "ZFITTER An Analytical Program for Fermion Pair Production in $e^{+} e^{-}$Annihilation", hep-ph/9412201 (1994).

[7] DELPHI Collaboration, P. Aarnio et al., Nucl. Inst. Meth. A303 (1991) 233; DELPHI Collaboration, P. Abreu et al., CERN-PPE/95-194, subm. to Nucl. Inst. Meth. A.

[8] DELPHI Collaboration, P. Abreu et al., Nucl. Phys. B418 (1994) 403.

[9] J.E. Campagne \& R. Zitoun, Z. Phys. C43 (1989) 469. 